\title{
Collider prospects for the muon $g-2$ in a general two-Higgs-doublet model
}

\author{
Wei-Shu Hou $\odot,{ }^{1}$ Rishabh Jain $\odot,{ }^{1}$ Chung Kao $\odot,{ }^{2}$ Girish Kumar $\odot,{ }^{1}$ and Tanmoy Modak $^{3}$ \\ ${ }^{1}$ Department of Physics, National Taiwan University, Taipei 10617, Taiwan \\ ${ }^{2}$ Homer L. Dodge Department of Physics and Astronomy, University of Oklahoma, \\ Norman, Oklahoma 73019, USA \\ ${ }^{3}$ Institut für Theoretische Physik, Universität Heidelberg, 69120 Heidelberg, Germany
}

(Received 3 July 2021; accepted 1 October 2021; published 27 October 2021)

\begin{abstract}
Recent progress on muon $g-2$ measurement prompts one to take it even more seriously. In the general two Higgs doublet model that allows extra Yukawa couplings, we take a simplified approach of single enhanced coupling. We fix the charged lepton flavor violating coupling, $\rho_{\tau \mu}=\rho_{\mu \tau}$, via the one-loop mechanism, for illustrative masses of the heavy scalar $H$ and pseudoscalar $A$, where we assume $m_{A}=m_{H^{+}}$. Since extra top Yukawa couplings are plausibly the largest, we turn on $\rho_{t t}$ and find that LHC search for $g g \rightarrow H, A \rightarrow \tau \mu$ gives a more stringent bound than from $\tau \rightarrow \mu \gamma$ with the two-loop mechanism. Turning on a second extra top Yukawa coupling, $\rho_{t c}$, can loosen the bound on $\rho_{t t}$, but LHC constraints can again be more stringent than from $B \rightarrow D \mu \nu$ versus $D e \nu$ universality. This means that evidence for $H, A \rightarrow \tau \mu$ may yet emerge with full LHC Run 2 data, while direct search for $\tau^{ \pm} \mu^{\mp} b W^{+}$or $t \bar{c} b W^{+}$(plus conjugate) may also bear fruit.
\end{abstract}

DOI: $10.1103 /$ PhysRevD.104.075036

\section{INTRODUCTION}

After extended meticulous efforts, the Fermilab Muon $g-2$ experiment announced recently their first measurement $[1], a_{\mu}($ FNAL $)=116592040(54) \times 10^{-11}(0.46 \mathrm{ppm})$. This confirms the previous result [2] at Brookhaven National Laboratory, combining to give [1]

$$
a_{\mu}(\operatorname{Exp})=116592061(41) \times 10^{-11}(0.35 \mathrm{ppm}) .
$$

Comparing this with the "consensus" theory prediction [3] for the Standard Model (SM), namely $a_{\mu}(\mathrm{SM})=$ $116591810(43) \times 10^{-11}(0.37 \mathrm{ppm})$, the difference,

$$
a_{\mu}(\operatorname{Exp})-a_{\mu}(\mathrm{SM})=(251 \pm 59) \times 10^{-11},
$$

is at $4.2 \sigma$. Equation (1), however, is consistent with a new lattice result [4] based on staggered fermions. Thus, the issue of the true SM value remains. We shall take Eq. (2) as is and seek $1 \sigma$ solution with new physics.

The persistence of the "muon $g-2$ anomaly" means there is a truly vast theory literature; hence, we refer to a very recent comprehensive account [5] for more complete references. Reference [5] stresses the need for chiral

Published by the American Physical Society under the terms of the Creative Commons Attribution 4.0 International license. Further distribution of this work must maintain attribution to the author(s) and the published article's title, journal citation, and DOI. Funded by SCOAP ${ }^{3}$. enhancement in solving the muon $g-2$ anomaly, whereas the two Higgs doublet model (2HDM) is "the only possibility without introducing new vector bosons or leptoquarks." We shall follow the 2HDM that is "not flavor aligned" [5], which possesses extra Yukawa couplings such as charged lepton flavor violating (CLFV) $\tau-\mu$ couplings, namely Ref. [6] (which descends from Refs. [7-12]). We expand on the impact at the Large Hadron Collider (LHC) by considering extra top Yukawa couplings [13].

The well-known 2HDM Models I and II invoke a $Z_{2}$ symmetry to implement the Natural Flavor Conservation (NFC) condition of Glashow and Weinberg [14], i.e., just one Yukawa matrix per quark charge (and for charged leptons as well). However, this is "special," if not ad hoc, so in the general 2HDM (g2HDM), one drops the $Z_{2}$ symmetry and lets nature reveal her flavor design. First called Model III [15], and following the footsteps of the ChengSher ansatz [16], indeed, the emergent fermion mass mixing hierarchies can be exploited to ease the worries [14] of flavor changing neutral couplings (FCNC): Extra Yukawa matrices should trickle off when going off-diagonal. The recent emergent alignment phenomenon, that the observed $h$ boson at $125 \mathrm{GeV}$ resembles very closely [17] the SM Higgs boson, brought in a flavor-independent surprise: alignment suppresses [18] FCNC involving the $h$ boson. Nature's designs for flavor seem intricate.

The alignment control of FCNC is illustrated by $h \rightarrow \tau \mu$ search. The Compact Muon Solenoid (CMS) experiment found initially [19] an intriguing $2 \sigma$ hint, which subsequently disappeared [20]. The full Run 2 data at $13 \mathrm{TeV}$ gives [21] 


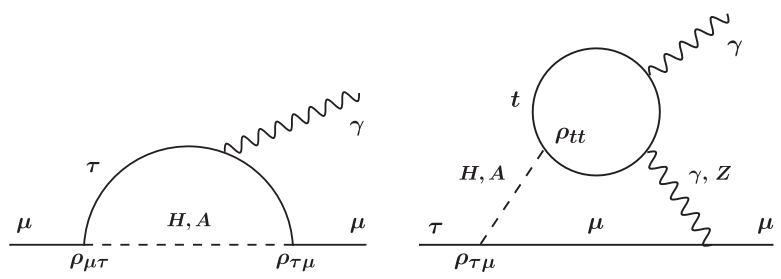

FIG. 1. One-loop mechanism for muon $g-2$ and two-loop diagram for $\tau \rightarrow \mu \gamma$.

$$
\mathcal{B}(h \rightarrow \tau \mu)<0.15 \% . \quad(\mathrm{CMS} 2021)
$$

but since the FCNC $\rho_{\tau \mu}$ arises from the heavy exotic doublet $\Phi^{\prime}\left(\left\langle\Phi^{\prime}\right\rangle=0\right)$, rather than the mass-giving doublet $\Phi$ (sole source of vacuum expectation value), the $h \tau \mu$ couples as $\rho_{\tau \mu} c_{\gamma}$, where $c_{\gamma} \equiv \cos \gamma$ is the $h-H$ mixing angle between the two $C P$-even scalar bosons. Thus, the alignment that $c_{\gamma}$ or $h-H$ mixing is small, can account for Eq. (3) without requiring $\rho_{\tau \mu}$ to be small, which is analogous [22] to another FCNC process, $t \rightarrow c h$ (with coupling $\rho_{t c} c_{\gamma}$ ). This is the starting point for a one-loop mechanism [see Fig. 1 (left)] to account for the muon $g-2$ anomaly $[9,10]$, originally stimulated by the CMS hint [19] for $h \rightarrow \tau \mu$. References [6,11] followed up on further LHC implications.

Invoking the one-loop mechanism of Fig. 1 (left) to account for muon $g-2$ for $m_{A, H}$ at the weak scale implies rather large $\rho_{\tau \mu}$ [6-11] at several tens times $\lambda_{\tau} \cong 0.01$ the tau Yukawa coupling in SM. Equation (3) then demands $\left|c_{\gamma}\right| \ll 1$, i.e., near the alignment limit. On one hand, this calls for a symmetry, which we do not get into. On the other hand, one should turn to $H, A \rightarrow \tau \mu$ (and $\left.H^{+} \rightarrow \tau^{+} \nu_{(\mu)}, \mu^{+} \nu_{(\tau)}\right)$ search [23], as it is not hampered by small $c_{\gamma}$ but at full strength of $s_{\gamma}(\equiv \sin \gamma) \rightarrow-1$. This needs finite $\rho_{t t}$ for gluon-gluon fusion production, so let us articulate our approach.

With one Higgs doublet of the SM already fully affirmed, adding a second doublet is the most conservative and simple extension. But, while simple, without a $Z_{2}$ symmetry to enforce NFC, the g2HDM possesses many new parameters. We therefore take a simplified approach of one large extra Yukawa coupling at a time, especially if it is greatly enhanced compared with analogous SM couplings. By analogy with the known top Yukawa coupling, $\lambda_{t} \cong 1$, however, it seems plausible that the extra top Yukawa coupling $\rho_{t t}$ is the strongest. Taking $\rho_{\tau \tau} \lesssim \mathcal{O}\left(\lambda_{\tau}\right)$ to keep the one-loop effect small, the large $\rho_{\tau \mu}$ needed for muon $g-2$ can induce $\tau \rightarrow \mu \gamma$ with finite $\rho_{t t}$ [8] through the two-loop diagram of Fig. 1 (right), which places a bound on $\rho_{t t} \rho_{\tau \mu}$, where the Belle experiment has recently updated [24] with full data. This constrains $\rho_{t t}$ to be considerably smaller than the $\rho_{\tau \mu}$ needed for muon $g-2$. We will show that a recent search [25] for $g g \rightarrow H \rightarrow \tau \mu$ by CMS with $36 \mathrm{fb}^{-1}$ data at $13 \mathrm{TeV}$, when interpreted in $\mathrm{g} 2 \mathrm{HDM}$, would place bounds on $\rho_{t t}$ that are more stringent than from Belle.
To enlarge the allowed range for $\rho_{t t}$, we turn on a second extra top Yukawa coupling, $\rho_{t c}$, which can dilute [6] the branching ratio $\mathcal{B}(H, A \rightarrow \tau \mu)$, thereby extending the allowed range for $\rho_{t t}$. However, the product of $\rho_{t c} \rho_{\tau \mu}$ can induce $B \rightarrow D \mu \nu_{\tau}$ through $H^{+}$exchange and affect the measured $B \rightarrow D \mu \nu$ rate (as the $\nu_{\tau}$ flavor cannot be detected), thereby breaking universality [6] with $B \rightarrow D e \nu$. Depending on the $\mathrm{H}^{+}$mass, $\rho_{t c}$, comparable in strength to $\rho_{\tau \mu}$, can be allowed. We will argue that searching for $\tau^{ \pm} \mu^{\mp} b W^{+}$or $t \bar{c} b W^{+}$at the LHC should be of interest.

The purpose of this paper is therefore threefold. First, g2HDM can [6-11] account for the muon $g-2$ anomaly, which is not new. Second, possible astounding signatures may emerge soon, not just at Belle II, but also at the LHC if large $\rho_{\tau \mu}$ is behind the muon $g-2$ anomaly. Our two LHC contact points above imply that $g g \rightarrow H, A \rightarrow \tau \mu$ may suddenly emerge, perhaps even with full Run 2 data, or detailed work may discover novel signatures such as $\tau^{ \pm} \mu^{\mp} b W^{+}$or $t \bar{c} b W^{+}$at the LHC. Third, this illustrates how limited our knowledge of g2HDM really is.

In Sec. II, we discuss the one-loop mechanism and find the bound on $\rho_{\tau \mu}$ and then discuss flavor constraints on $\rho_{t t}$ and $\rho_{t c}$ that follow, as well as various flavor concerns; in Sec. III, we compare $\tau \rightarrow \mu \gamma$ constraint on $\rho_{t t}$ with direct search for $g g \rightarrow H, A \rightarrow \tau \mu$ and $B \rightarrow D \ell \nu$ universality $(\ell=e, \mu)$ constraint on $\rho_{t c}$ with $\tau^{ \pm} \mu^{\mp} b W^{+}$or $t \bar{c} b W^{+}$ search at the LHC; after some discussion in Sec. IV, we offer our summary.

\section{ONE-LOOP MECHANISM FOR MUON $g-2$}

The Yukawa couplings in g2HDM are [26,27]

$$
\begin{aligned}
& -\bar{\nu}_{i} \rho_{i j}^{\ell} R \ell_{j} H^{+}-\bar{u}_{i}\left[\left(V \rho^{d}\right)_{i j} R-\left(\rho^{u \dagger} V\right)_{i j} L\right] d_{j} H^{+} \\
& -\frac{1}{\sqrt{2}} \sum_{f=\ell}^{u, d} \bar{f}_{i}\left[\left(\lambda_{i}^{f} \delta_{i j} c_{\gamma}+\rho_{i j}^{f} s_{\gamma}\right) H-i \operatorname{sgn}\left(Q_{f}\right) \rho_{i j}^{f} A\right. \\
& \left.-\left(\lambda_{i}^{f} \delta_{i j} s_{\gamma}-\rho_{i j}^{f} c_{\gamma}\right) h\right] R f_{j}+\text { H.c. },
\end{aligned}
$$

where $i, j$ are summed over generations, $L, R=(1 \mp$ $\left.\gamma_{5}\right) / 2$ are projection operators, and $V$ is the CKM matrix, with the lepton matrix taken as unity due to vanishing neutrino masses. One can therefore read off the $\rho_{\mu \tau}, \rho_{\tau \mu}$ and $\rho_{t t}$ couplings indicated in Fig. 1.

We do not write down the Higgs potential $V\left(\Phi, \Phi^{\prime}\right)$ (except assuming it is $C P$ conserving), as it can be found in many papers traced to Ref. [26]. We shall take $m_{H}=$ $300 \mathrm{GeV}$ as a benchmark to keep $\rho_{\tau \mu}$ "reasonable" and illustrate with $m_{A}=m_{H^{+}}=340,420,500 \mathrm{GeV}$. That is, we assume custodial symmetry (namely $\eta_{4}=\eta_{5}$, in the notation of Ref. [18]) to reduce tension with oblique parameter constraints. We follow, for example, Ref. [28] and perform a parameter scan utilizing 2HDMC [29] to demonstrate that there is a parameter space that satisfies 


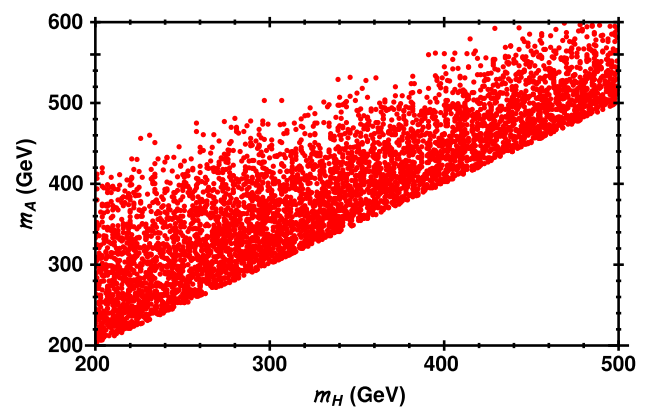

FIG. 2. Parameter space in $m_{H}-m_{A}$ that satisfies perturbativity, unitarity, positivity, and oblique electroweak constraints.

perturbativity, unitarity, and positivity, as well as precision electroweak constraints, as shown in Fig. 2. The scan is performed with $m_{H}=[200,500] \mathrm{GeV}, m_{A}=m_{H^{+}}=$ $[200,600] \mathrm{GeV}, \eta_{2}=[0,5]$ (as required by positivity), $\eta_{3}=[-5,5]$, and $\eta_{7}=[-5,5]$, and setting the alignment limit $\cos \gamma=0$ with $m_{A}>m_{H}$.

As we will soon see, to provide $1 \sigma$ solution to the muon $g-2$ anomaly for benchmark $m_{H}, m_{A}$ values, $\rho_{\tau \mu}=\rho_{\mu \tau}$ (which we take to simplify), need to be $\sim 20$ times the strength of $\lambda_{\tau} \simeq 0.01$. Equation (3) then implies

$$
\left|\rho_{\tau \mu} c_{\gamma}\right| \lesssim 0.1 \lambda_{\tau}
$$

So, $\rho_{\tau \mu} \sim 0.2$ gives $c_{\gamma} \lesssim 0.005$, which is close to the alignment limit. In this paper, we take the alignment limit of $c_{\gamma} \rightarrow 0$ and $s_{\gamma} \rightarrow-1$ to simplify.

Setting $c_{\gamma}=0$ and using the well-known one-loop formula [6-11], we plot $\Delta a_{\mu}$ vs $\rho_{\tau \mu}=\rho_{\mu \tau}$ for $m_{H}=$ $300 \mathrm{GeV}$ and $\Delta m=m_{A}-m_{H}=10,40,120,200 \mathrm{GeV}$ in Fig. 3, together with the $1 \sigma$ and $2 \sigma$ ranges from Eq. (2). We note that $A$ and $H$ exactly cancel one another in $\Delta a_{\mu}$ when degenerate, which is illustrated by $\Delta m=$ $10 \mathrm{GeV}$. Such near degenerate masses would require extremely large $\rho_{\tau \mu}$ values. With $m_{H}=300 \mathrm{GeV}$ as

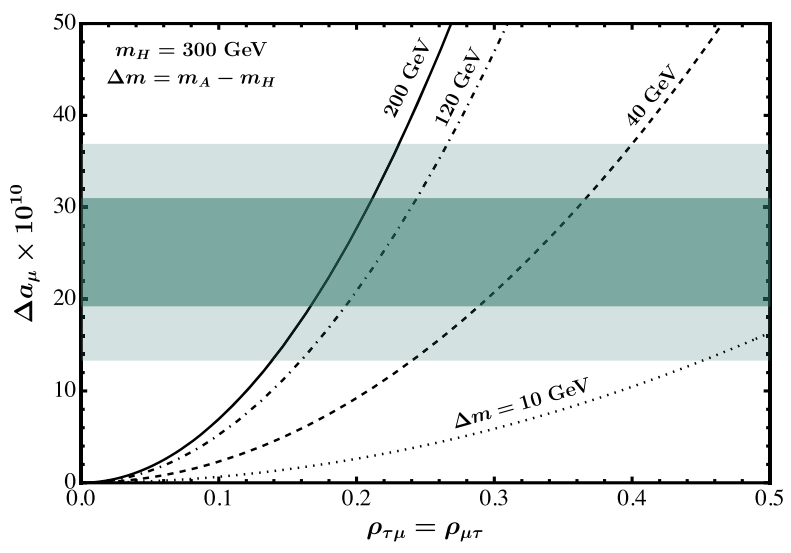

FIG. 3. $\Delta a_{\mu}$ versus $\rho_{\tau \mu}=\rho_{\mu \tau}$ for $m_{H}=300 \mathrm{GeV}$ and $\Delta m=m_{A}-m_{H}=10,40,120,200 \mathrm{GeV}$, with experimental $1 \sigma$ and $2 \sigma$ ranges [Eq. (2)] as indicated. benchmark, the three values of $m_{A}=340,420,500 \mathrm{GeV}$ are taken for illustration. One sees that, as $m_{A}$ increases toward decoupling, $H$ starts to dominate and the $\rho_{\tau \mu}$ strength needed for $1 \sigma$ solution decreases, namely

$$
\rho_{\tau \mu} \gtrsim 0.289,0.192,0.167,
$$

which are $\sim 20$ times larger than $\lambda_{\tau} \simeq 0.01$.

If one replaces the muon in the left of Fig. 1 (left) by a tau, a finite $\rho_{\tau \tau}$ can induce $\tau \rightarrow \mu \gamma$. We have checked that for $\rho_{\tau \tau}=\mathcal{O}\left(\lambda_{\tau}\right)$ or smaller [30], the one-loop chiral suppression gives a rate that is quite below existing bounds 20]]. It is known [8] that a two-loop mechanism can, in fact, dominate for appropriate $\rho_{t t}$ values in the fermion loop, as seen from Fig. 1 (right). The mechanism is similar to $\mu \rightarrow e \gamma$ [31], from which the formulas are adapted. Using the known formulas and $1 \sigma$ solution values of Eq. (6), and assuming $\rho_{\tau \tau} \sim 0$, one finds $\left|\rho_{t t} \rho_{\tau \mu}\right|<0.014,0.016,0.017$ for $m_{H}=300 \mathrm{GeV}$ and $m_{A}=340,420,500 \mathrm{GeV}$, or

$$
\left|\rho_{t t}\right| \lesssim 0.05,0.08,0.1
$$

which are rather small compared with $\lambda_{t} \cong 1$. With $\rho_{t t}$ considerably smaller than 1 , it is generally easier to accommodate flavor constraints, especially in the quark sector [32,33], and we do not explore it further.

As we will show in the next section, the search for $g g \rightarrow$ $H, A \rightarrow \tau \mu$ at the LHC turns out to provide more stringent bound on $\rho_{t t}$ than Eq. (7). One thought [6] then is to dilute $\mathcal{B}(H, A \rightarrow \tau \mu)$ with a second extra top Yukawa coupling, namely $\rho_{t c}$. However, $B \rightarrow D \mu \nu$ vs $B \rightarrow D e \nu$ universality provides a bound [6] on $\rho_{t c} \rho_{\tau \mu}$ through $H^{+}$exchange, where the $H^{+} \bar{c} b$ and $H^{+} \mu \bar{\nu}_{\tau}$ couplings can be read off Eq. (4). The not so intuitive point is that the former is $\simeq V_{t b} \rho_{t c}[34]$ and not CKM suppressed. Thus, $\rho_{t c} \rho_{\tau \mu}$ would generate $B \rightarrow D \mu \bar{\nu}_{\tau}$, which would add incoherently [11] to $B \rightarrow D \mu \bar{\nu}$ that is measured by experiment rather precisely, but cannot discern the neutrino flavor. From Eq. (6) and using Eq. (84) of Ref. [11], we compare with the Belle measurement [35],

$\mathcal{B}(B \rightarrow D \mu \nu) / \mathcal{B}(B \rightarrow D e \nu)=0.995 \pm 0.022 \pm 0.039$.

Adding errors in quadrature and allowing $2 \sigma$ range, we find $\left|\rho_{t c} \rho_{\tau \mu}\right| \lesssim 0.024,0.037,0.053$ for $m_{H}=300 \mathrm{GeV}$ and $m_{H^{+}}=m_{A}=340,420,500 \mathrm{GeV}$, giving

$$
\left|\rho_{t c}\right| \lesssim 0.08,0.19,0.32
$$

The decreasing value of $\rho_{\tau \mu}$ with increasing $m_{A}$, namely Eq. (6), implies the opposite for $\rho_{t c}$, which for $m_{A} \gtrsim$ $400 \mathrm{GeV}$, can surpass the $\rho_{\tau \mu}$ strength needed for $1 \sigma$ solution of muon $g-2$. 
In fact, a good part of the dilution effect for $\mathcal{B}(H, A \rightarrow$ $\tau \mu)$ is driven by the opening of $A \rightarrow H Z$ decay for $m_{A} \gtrsim 400 \mathrm{GeV}$, which is one reason why we increased $\Delta m=m_{A}-m_{H}>m_{Z}$. With $m_{A}=m_{H^{+}}$, this means $H^{+} \rightarrow H W^{+}$also opens up. As we shall see in the next section, a sizable $\rho_{t c}$ can give rise to $c g \rightarrow b H^{+}$production [34] (again, the $\bar{c} b H^{+}$coupling is not CKM-suppressed), as well as [36] $c g \rightarrow t H, t A$, with $c g \rightarrow t A$ suppressed for higher $m_{A} \cdot H^{+} \rightarrow H W^{+}$decay with $H \rightarrow t \bar{c}, \tau \mu$ would lead to additional signatures at the LHC.

Let us comment on a few other flavor concerns. With $\rho_{\tau \mu}$ sizable, it can induce $\tau \rightarrow 3 \mu$ decay at tree level with $\rho_{\mu \mu} \neq 0$. Thus, the bound by Belle [37], $\mathcal{B}(\tau \rightarrow 3 \mu) \lesssim$ $2.1 \times 10^{-8}$, puts a constraint on $\rho_{\mu \mu}$. Using formulas from Refs. [33,38], we find $\rho_{\tau \mu} \rho_{\mu \mu} \lesssim(240-320) \lambda_{\tau} \lambda_{\mu}$ for $m_{H}=$ $300 \mathrm{GeV}$ and $m_{A}-m_{H}=10-200 \mathrm{GeV}$. This implies $\rho_{\mu \mu} \lesssim 10 \lambda_{\mu}<0.01$, which is still rather small. One evades the $h \rightarrow \mu \mu$ search in alignment limit, $c_{\gamma}=0$, while one also evades the recent $H, A \rightarrow \mu \mu$ search [39] by CMS after the multimode dilution effects discussed in the next section are taken into account.

The coupling $\rho_{\mu \tau}\left(\rho_{\tau \mu}\right)$ enters the $H^{+} \bar{\nu}_{\mu} \tau\left(H^{+} \bar{\nu}_{\tau} \mu\right)$ coupling directly and can generate $\tau \rightarrow \mu \nu_{\mu} \bar{\nu}_{\tau}$, where the neutrino flavors are swapped compared with $W$ boson exchange, which the experiment cannot distinguish. Compared with $\tau(\mu) \rightarrow e \nu \bar{\nu}$, this constitutes another test of lepton universality violation at the per mille level, which has been recorded by HFLAV [40]. We have checked that $\rho_{\tau \mu}=\rho_{\mu \tau} \lesssim 0.9$ is still allowed for our benchmark masses, which is more accommodating than muon $g-2$.

Finally, the $\rho_{\tau \mu}=\rho_{\mu \tau}$ coupling affects $Z \rightarrow \tau \tau, \mu \mu$ decays, which have been precisely measured [20], again at the per mille level. We find the bound to be weaker than the bounds from $\tau$ decays discussed here.

\section{FLAVOR PROBES VS LHC SEARCH}

The connection between muon $g-2$ (or $\tau \rightarrow \mu \gamma$ ) with the $H, A \rightarrow \tau \mu$ search [6-11] at hadron colliders is well known. In part because of the $\tau \rightarrow \mu \gamma$ constraint on $\rho_{t t}$, Ref. [6] did not explore $g g \rightarrow H, A \rightarrow \tau \mu$ but emphasized the electroweak pair production of exotic scalar boson pairs instead. However, we are interested in keeping $\rho_{t t}$ as CMS has recently performed a search for the heavy $H \rightarrow \tau \mu$ (and $\tau e$ ) channel. Using $\sim 36 \mathrm{fb}^{-1}$ data at $13 \mathrm{TeV}$ and assuming narrow width, the bound [25] on $\sigma(g g \rightarrow H) \mathcal{B}(H \rightarrow \tau \mu)$ ranges from $51.9 \mathrm{fb}$ to $1.6 \mathrm{fb}$ for $m_{H}$ ranging from $200 \mathrm{GeV}$ to $900 \mathrm{GeV}$. The $H$ notation is intentional: CMS followed Ref. [41] and studied the scalar $H$ case, but not for pseudoscalar $A$.

It is of interest to check the current LHC bound on $\rho_{t t}$ within the large $\rho_{\tau \mu}=\rho_{\mu \tau}$ interpretation of muon $g-2$. We evaluate the $p p \rightarrow H, A \rightarrow \tau \mu+X$ cross section for LHC at $13 \mathrm{TeV}$, assuming $g g \rightarrow H, A$ as the dominant subprocess, and convolute with parton distribution functions. Taking $\rho_{t t}$ as real for our benchmark $m_{H}$ and $m_{A}$ values, we fold in the decay branching ratios $\mathcal{B}(H, A \rightarrow \tau \mu)$ and give our results in Fig. 4 as blue (red) solid curves for $H$ $(A)$, with the corresponding CMS bound given as horizontal lines for our three benchmark sets. The bounds from $\tau \rightarrow \mu \gamma$, Eq. (7), are given as (dark pink) vertical lines. We have checked that $\Gamma_{H} \sim 1 \mathrm{GeV}$, while $\Gamma_{A}<2 \mathrm{GeV}$ for $m_{A}<400 \mathrm{GeV}$, and remains below $10 \mathrm{GeV}$ for $m_{A} \sim 500 \mathrm{GeV}$, hence, satisfying the narrow width approximation. We do not combine the two separate states.

We see that, with only a subset of Run 2 data, the CMS bound is more stringent than the bound from $\tau \rightarrow \mu \gamma$, as one should always take the more stringent bound out of $H$ vs $A$. For the cases of $m_{A}=340,420 \mathrm{GeV}$, the larger production cross sections for $A$ imply a more stringent limit than from
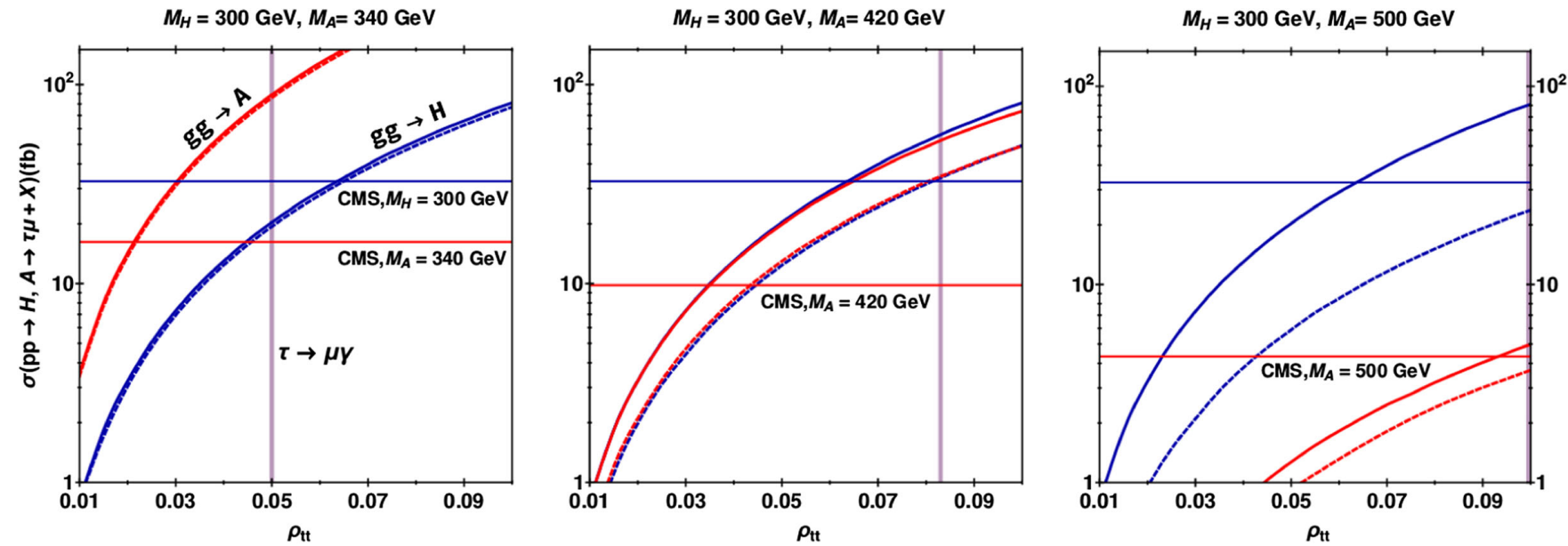

FIG. 4. Cross sections for $p p \rightarrow H(A) \rightarrow \tau \mu+X$ vs $\rho_{t t}$ at $\sqrt{s}=13 \mathrm{TeV}$, where blue (red) solid curves are for $\rho_{\tau \mu} \simeq 0.289$ (left), 0.19 (center), 0.167 (right) from Eq. (6) with $\rho_{t c}=0$ and $m_{H}=300 \mathrm{GeV}$, and for $m_{A}=340,420,500 \mathrm{GeV}$, respectively. The dashed curves correspond to $\rho_{t c} \simeq 0.08$ (left), 0.19 (center), and 0.32 (right) from Eq. (8). All other $\rho_{i j}^{f}$ s are set to zero. Bounds from $\tau \rightarrow \mu \gamma$ [24] and CMS search [25] for $g g \rightarrow H \rightarrow \tau \mu$ (reinterpreted in case of $A$ ) are indicated. 
$H$, but for $m_{A}=500 \mathrm{GeV}$, parton densities have dropped too low, and the bound from $H$ is more stringent than $A$, but still more stringent than from $\tau \rightarrow \mu \gamma$. We remark that the case of $m_{H}, m_{A}=300,340 \mathrm{GeV}$, i.e., Fig. 4 (left), does provide a $1 \sigma$ solution to muon $g-2$, but if all other extra Yukawa couplings, $\rho_{i j}^{f} \mathrm{~s}(f=u, d, \ell)$, are not stronger than $\lambda_{\max (\mathrm{i}, \mathrm{j})}$ the Yukawa strength in SM, then there are little other consequences, except in the $g g \rightarrow A \rightarrow \tau \mu$ probe of $\rho_{t t}$ itself. The reinterpreted CMS bound of $\rho_{t t} \lesssim 0.02$ from Fig. 4 (left) is below $\rho_{t t} \lesssim 0.05$, allowed by $\tau \rightarrow \mu \gamma$, which is really small compared with $\lambda_{t} \cong 1$. There is still discovery potential with full Run 2 data. The allowed range for $\rho_{t t}$ from CMS gets partially restored for heavier $m_{A}$, where $g g \rightarrow H, A \rightarrow \tau \mu$ production becomes predominantly through $H$ in Fig. 4 (right). The easing of the bound on $\rho_{t t}$ is due to the opening of $A \rightarrow t \bar{t}, H Z$, as can be seen from the branching ratio plot, Fig. 5 (left).

In addition to $\rho_{t t}$ needed for $\tau \rightarrow \mu \gamma$ and $g g \rightarrow H, A$ production, we give the effect of turning on $\rho_{t c}$, the second extra top Yukawa coupling, in Fig. 5 (left), as it can dilute the $g g \rightarrow H, A \rightarrow \tau \mu$ cross section. However, as discussed in the previous section, the product of $\rho_{t c} \rho_{\tau \mu}$ can make $B \rightarrow D \mu \nu$ deviate from $B \rightarrow D e \mu$ and break $e-\mu$ universality. From the $1 \sigma$ solution to muon $g-2$, Eq. (6), for our benchmark, $m_{H}=300 \mathrm{GeV}, m_{A}=340,420,500 \mathrm{GeV}$, Belle data [35] therefore leads to the bound on $\rho_{t c}$, giving Eq. (8). Taking $\rho_{t c} \simeq 0.08,0.19,0.32$, and the $\rho_{t c}$-induced dilution for the three corresponding $m_{A}$ values in Fig. 5 (left), we draw the dashed curves in Fig. 4. Not much is changed in Fig. 4 (left), but for Fig. 4 (center) and Fig. 4 (right), the $g g \rightarrow H, A \rightarrow \tau \mu$ cross section weakens progressively. It is less significant for $A$ production at $m_{A}=$ $500 \mathrm{GeV}$ in Fig. 4 (right) because $A \rightarrow H Z$ is much stronger, but the corresponding drop in $g g \rightarrow H \rightarrow \tau \mu$ is rather significant. This is because the $\rho_{t c} \simeq 0.32$ value is about twice as large as the $\rho_{\tau \mu}$ value of $\simeq 0.167$. The large $\rho_{t c}$ brings about interest, and thereby possible constraints, from production processes at the LHC.

Before discussing $\rho_{t c}$-induced processes, we remark that Fig. 5, where we also give $H^{+}$decay branching fractions, is not just for our three benchmark values of $m_{A}=340,420,500 \mathrm{GeV}$. The plot is made by the same approach: For $m_{H}=300 \mathrm{GeV}$ and a given $m_{A}$, we find the $1 \sigma$ muon $g-2$ solution value for $\rho_{\tau \mu}$ analogous to Eq. (6), then find the allowed upper bounds for $\rho_{t t}$ and $\rho_{t c}$, analogous to Eqs. (7) and (9), respectively. The plot therefore scans through $m_{A}$, with $m_{H}$ fixed at $300 \mathrm{GeV}$.

Having $\rho_{t c}$ alone with $\rho_{t t}$ small, it can generate [36] $c g \rightarrow t H, t A \rightarrow t t \bar{c}$ or same-sign top plus jet final state, which can [42] feed the $t \bar{t} W$ control region (CRW) of $4 t$ search by CMS (by different selection cuts, ATLAS is less stringent), where there is now a full Run 2 data study [43]. More significant is a study [28] of $c g \rightarrow b H^{+} \rightarrow b A W^{+}$ production followed by $A \rightarrow t \bar{c}$ and both the top and the $W^{+}$decay (semi)leptonically, which can also feed the CRW of CMS $4 t$ search. In this study, the $H^{+} \rightarrow$ $A W^{+}$decay was treated with $m_{H^{+}}=m_{H}$, i.e., swapping $H \leftrightarrow A$ from our present case. The $b H^{+}$cross section is sizable due to a light $b$ quark and no CKM suppression for the $\bar{c} b H^{+}$coupling [34]. It was found that $\rho_{t c} \sim 0.15$ is barely allowed for $m_{H^{+}} \sim 500 \mathrm{GeV}$. This may seem to make the large values of $\rho_{t c}$ for our dilution effect untenable.

However, the dilution effect may solve itself. We note that, assuming just two associated extra Yukawa couplings, i.e., $\rho_{\tau \mu}$ and $\rho_{t c}$, the two branching ratios are $\mathcal{B}(H \rightarrow t \bar{c}+\bar{t} c): \mathcal{B}(H \rightarrow \tau \mu)=5 \%: 95 \%, 40 \%: 60 \%$, $71 \%: 29 \%$, for $m_{A}=340,420,500 \mathrm{GeV}$ and $\left(\rho_{\tau \mu}, \rho_{t c}\right)=$ $(0.289,0.08),(0.192,0.19),(0.167,0.32)$, respectively. For the $m_{A}=m_{H^{+}}=340 \mathrm{GeV}$ benchmark, $\rho_{t c}$ is too small to be of concern. Likewise, for the $m_{A}=m_{H^{+}}=420 \mathrm{GeV}$ benchmark, from Fig. 4 of Ref. [13], which is for $m_{H}=$ $300 \mathrm{GeV}$ and $m_{A}=350 \mathrm{GeV}$, the CRW of CMS $4 t$ search would push $\rho_{t c}$ to below 0.4. However, $m_{A}$ at the much heavier $420 \mathrm{GeV}$ reduces $c g \rightarrow t A \rightarrow t t \bar{c}$ events that feed $\mathrm{CRW}$, so one is not yet sensitive to $\rho_{t c} \simeq 0.2$, while there is some dilution from $A \rightarrow H Z$. For the more efficient $c g \rightarrow$ $b H^{+}$production, we see from Fig. 5 (right) that $H^{+} \rightarrow c \bar{b}$ at $\simeq 32 \%$ is pure dilution, and even $\tau \nu_{(\mu)}+\mu \nu_{(\tau)}$ at $\simeq 22 \%$ together with the $b$, would likely be overwhelmed by single-top background. The dominant $(\simeq 41 \%) b H W^{+}$final state would be $20 \% b t \bar{c} W^{+}$and $60 \% b \tau^{ \pm} \mu^{\mp} W^{+}$. The latter would be an interesting signature in itself, but would not feed CRW of CMS $4 t$ search.

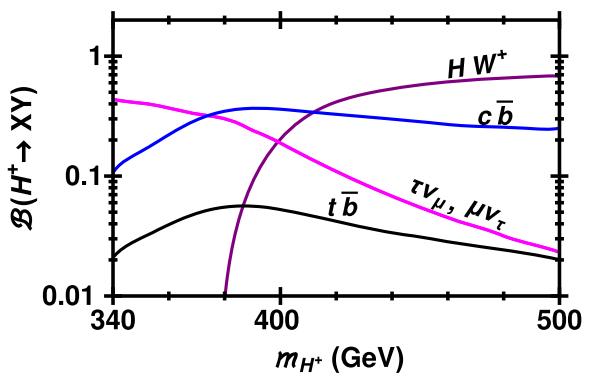

FIG. 5. Branching fractions for $A$ and $H^{+}$decay modes versus mass from $\rho_{\tau \mu}, \rho_{t c}, \rho_{t t}$ [see Eq. (4)] and the weak interaction, where $m_{H}=300 \mathrm{GeV}$ and $m_{A}=m_{H^{+}}$is assumed. 
It is the $m_{A}=m_{H^{+}}=500 \mathrm{GeV}$ benchmark that CRW of CMS $4 t$ may put a limit on $\rho_{t c}: H^{+} \rightarrow c \bar{b}$ is slightly below $30 \%$, while $\tau \nu_{(\mu)}+\mu \nu_{(\tau)}$ is no longer a dilution factor. This is due to the dominance of $\mathrm{H}^{+} \rightarrow H W^{+}$, now at $70 \%$. With $H \rightarrow t \bar{c}$ at $\sim 35 \%$, it would feed $4 t$ CRW abundantly, as pointed out in Ref. [28] for the analogous twisted custodial case for $b A W^{+}$production. If we take the number there as a guesstimate, then $\rho_{t c} \sim 0.15$ would be borderline. This just means that $\rho_{t c}$ should be considerably less than 0.32 from Eq. (8), which means that one cannot reach $\rho_{t t} \sim 0.1$ as allowed by $\tau \rightarrow \mu \gamma$.

We note that, from Fig. 2 and for $m_{H} \sim 300 \mathrm{GeV}, m_{A}=$ $m_{H^{+}}$at $500 \mathrm{GeV}$ is already at the "border" of the scan space. Thus, we conclude that for large splitting of $m_{A}=$ $m_{H^{+}}$from $m_{H}$, the solution to muon $g-2$ would not allow a $\rho_{t c}$ value larger than $\rho_{\tau \mu}$ for the one-loop solution. As one moves $m_{H}$ higher, the discussion should be similar for higher $m_{A}=m_{H^{+}}$, requiring larger $\rho_{\tau \mu}$ values to solve muon $g-2$.

\section{DISCUSSION AND CONCLUSION}

Referring to the $4.2 \sigma$ "white space of disagreement" between theory and experiment, the question "What monsters may be lurking there?" from the April 7 announcement presentation by the Muon $g-2$ experiment became the quote of the day. We stress that $\rho_{\tau \mu} / \lambda_{\tau} \sim 20$, while large, is not "monstrous" and is up to nature to choose (and the counterbalancing $\rho_{t t} / \lambda_{t} \lesssim 1 / 10$ as well). This can be compared, for example, with $\tan \beta=\mathcal{O}\left(10^{3}\right)$ in the muon-specific 2HDM [44], tailor-made for the muon $g-$ 2 by some $Z_{4}$ symmetry. In a different $Z_{4}$ arrangement, the $\mu-\tau$-philic extra scalar doublet [45] was introduced with only the $\rho_{\tau \mu}=\rho_{\mu \tau}$ coupling, with further variations of [46] $m_{A}-m_{H}<50 \mathrm{GeV}$ by considering the $\sim 2 \sigma$ deviation from SM in $\tau$ decays or pushing $m_{H}$ to be very light [47]. We hope we have illustrated that such elaborations are not necessary and that the g2HDM is versatile enough with its arsenal of extra Yukawa couplings to provide solution to muon $g-2$ while hiding from view so far, but with promising LHC implications.

With enhanced $\rho_{\tau \mu}$ implied by muon $g-2$, it has been suggested $[48,49]$ that, by the natural complexity of extra Yukawa couplings, they could possibly drive electroweak baryogenesis (EWBG), i.e., be the $C P$-violating source of the baryon asymmetry of the Universe. In fact, part of our motivation for considering $\rho_{t t}$ and $\rho_{t c}$ are their potential as drivers [50] for EWBG. Our findings show that $\rho_{t t}$ may still suffice, but $\rho_{t c}$ may not be strong enough, but whether one deals with large $\rho_{\tau \mu}$ or small but finite $\rho_{t t}$ as the EWBG driver, one may have to face the issue of the alignment limit or extremely small $h-H$ mixing. We note that, by the arguments we have given, the simultaneously large $\rho_{\tau \mu}, \rho_{\tau \tau}$ and $\rho_{t t}$ as given in Ref. [48] cannot survive collider bounds such as from CMS search for $H, A \rightarrow \tau \mu$ [25], and ATLAS
[51] and CMS [52] searches for $H, A \rightarrow \tau \tau$, which we have checked explicitly.

We note in passing that g2HDM has been invoked in weaker (considered as a perturbation, hence, keeping $\tan \beta$ ) form [53], where having rather small $\rho_{\tau \mu}$ called for relatively light $m_{A}$. In a similar vein, a reinterpretation [54] of the CMS $g g \rightarrow H \rightarrow \tau \mu$ search [25] also urged for probing below $m_{H}=200 \mathrm{GeV}$. These suggestions are not invalid, but we chose $m_{H}$ within the search range of CMS at a typical weak scale to illustrate how g2HDM may resolve muon $g-2$.

The flavor conserving coupling $\rho_{\mu \mu}$ can, in principle, induce new contributions to muon $g-2$ via one- and two-loop diagrams. The one-loop effect [55] involves $\rho_{\mu \mu}$ only, but because of chiral suppression from muon in the loop, exorbitantly large $\rho_{\mu \mu} \gtrsim 0.85\left(\sim 1400 \lambda_{\mu}\right.$ : monstrous $)$ is needed for $1 \sigma$ solution to muon $g-2$ for the same benchmark masses as before. The two-loop effect [56,57], by replacing the $\tau$ on the left of Fig. 1 (right) with $\mu$, can be enhanced by extra top Yukawa coupling $\rho_{t t}$, but it again calls for the product of $\rho_{t t} \rho_{\mu \mu}$ to be too large. Taking, for example, $\rho_{t t} \sim-0.1$ for $m_{H}=300 \mathrm{GeV}, m_{A}=m_{H^{+}}=$ $340 \mathrm{GeV}$, one can achieve $1 \sigma$ solution to muon $g-2$ for $\rho_{\mu \mu} \gtrsim 0.42$, which is again rather large [58]. Indeed, this runs into conflict with the CMS search [39] (the ATLAS bound [59] at similar data size is weaker) for $g g \rightarrow H, A \rightarrow$ $\mu^{+} \mu^{-}$with $\sim 36 \mathrm{fb}^{-1}$, which restricts $\rho_{t t} \lesssim 10^{-2}$ for such a strong $\rho_{\mu \mu}$ value. This shows, by power of the CMS bound [39] on $g g \rightarrow H, A \rightarrow \mu^{+} \mu^{-}$even with just a fraction of LHC Run 2 data, that the two-loop $\rho_{\mu \mu}$ mechanism is not viable.

Finally, let us comment on the electroweak production processes suggested in Ref. [6], namely $p p \rightarrow H A, H^{ \pm} H$, $H^{ \pm} A, H^{+} H^{-}$through vector bosons. For large $\rho_{\tau \mu}$ values needed for muon $g-2$, dileptonic decays of $H, A, H^{ \pm}$ may prevail, which allow exquisite reconstruction [6] of exotic extra Higgs boson masses, hence, rather attractive. However, the electroweak production cross section, at the $\mathrm{fb}$ level, is rather small. Take the case of $m_{H}=300 \mathrm{GeV}$, $m_{A}=m_{H^{+}}=340 \mathrm{GeV}$, for example. If we allow the lowest $\rho_{t c}$ value of 0.08 in Eq. (9), nominally from $B \rightarrow$ $D \ell \nu$ universality constraint, we find that the $c g \rightarrow b H^{+}$ cross section, being a strong production process involving a gluon (with not too significant a suppression from charm parton distributions) and only one heavy particle in final state, is almost 2 orders of magnitude higher than the electroweak production processes. This effect persists for the other two benchmarks where $A$ and $H^{+}$are heavier, which allow for larger $\rho_{t c}$ values. Furthermore, as we have illustrated, multiple decay modes of the heavier $A$ and $H^{+}$(see Fig. 5), as well as $H \rightarrow \tau^{ \pm} \mu^{\mp}$, $t \bar{c}(\bar{t} c)$, would dilute one another, which works also for the decays of the extra scalar bosons in electroweak pair production. Thus, neither the search for the electroweak 
production of extra Higgs boson pairs, nor the reconstruction of the extra boson masses, may be as rosy as argued in Ref. [6].

In summary, we employ large CLFV Yukawa coupling $\rho_{\tau \mu}=\rho_{\mu \tau} \sim 0.2$ in the general 2HDM to account for muon $g-2$ anomaly through the known one-loop mechanism. The extra Higgs bosons have mass at the weak scale, but one is close to the alignment limit of very small $h-H$ mixing to evade $h \rightarrow \tau \mu$. This motivates the check with $g g \rightarrow H, A \rightarrow \tau \mu$ search, where a subset of LHC Run 2 data already puts a more stringent bound on the extra top Yukawa coupling $\rho_{t t}$ than from $\tau \rightarrow \mu \gamma$ through the twoloop mechanism. The stringent constraint on $\rho_{t t}$ can be eased by allowing a second extra top Yukawa coupling, $\rho_{t c}$, which motivates the search for $c g \rightarrow b H^{+} \rightarrow \tau^{ \pm} \mu^{\mp} b W^{+}$, $t \bar{c} b W^{+}$(same-sign dilepton plus two $b$-jets and additional jet, with missing $p_{T}$ ) at the LHC, on top of possible samesign top plus jet signatures from $c g \rightarrow t H, t A \rightarrow t t \bar{c}$. Whether these extra Yukawa couplings can drive electroweak baryogenesis should be further studied.

\section{ACKNOWLEDGMENTS}

The work of W. S. H. is supported by MOST 109-2112M-002-015-MY3 of Taiwan and NTU 110L104019 and 110L892101, and the work of R. J. and G. K. are supported by MOST 109-2811-M-002-565 and 109-2811-M-002540 , respectively. The research of C. K. was supported in part by the U.S. Department of Energy and the University of Oklahoma and T.M. by a postdoctoral research fellowship from the Alexander von Humboldt Foundation.
[1] B. Abi et al. (Muon g-2 Collaboration), Phys. Rev. Lett. 126, 141801 (2021).

[2] G. W. Bennett et al. (Muon g-2 Collaboration), Phys. Rev. D 73, 072003 (2006).

[3] T. Aoyama et al., Phys. Rep. 887, 1 (2020).

[4] S. Borsanyi et al., Nature (London) 593, 51 (2021).

[5] P. Athron, C. Balázs, D. H. Jacob, W. Kotlarski, D. Stöckinger, and H. Stöckinger-Kim, arXiv:2104.03691.

[6] S. Iguro, Y. Omura, and M. Takeuchi, J. High Energy Phys. 11 (2019) 130.

[7] K. A. Assamagan, A. Deandrea, and P. A. Delsart, Phys. Rev. D 67, 035001 (2003).

[8] S. Davidson and G. J. Grenier, Phys. Rev. D 81, 095016 (2010).

[9] Y. Omura, E. Senaha, and K. Tobe, J. High Energy Phys. 05 (2015) 028.

[10] Y. Omura, E. Senaha, and K. Tobe, Phys. Rev. D 94, 055019 (2016).

[11] S. Iguro and K. Tobe, Nucl. Phys. B925, 560 (2017).

[12] A. Crivellin, D. Müller, and C. Wiegand, J. High Energy Phys. 06 (2019) 119.

[13] For a brief review, see W.-S. Hou and T. Modak, Mod. Phys. Lett. A 36, 2130006 (2021).

[14] S. L. Glashow and S. Weinberg, Phys. Rev. D 15, 1958 (1977).

[15] W.-S. Hou, Phys. Lett. B 296, 179 (1992).

[16] T.-P. Cheng and M. Sher, Phys. Rev. D 35, 3484 (1987).

[17] G. Aad et al. (ATLAS and CMS Collaboration), J. High Energy Phys. 08 (2016) 045.

[18] W.-S. Hou and M. Kikuchi, Europhys. Lett. 123, 11001 (2018).

[19] V. Khachatryan et al. (CMS Collaboration), Phys. Lett. B 749, 337 (2015).

[20] P. A. Zyla et al. (Particle Data Group Collaboration), Prog. Theor. Exp. Phys. 2020, 083C01 (2020).
[21] A. M. Sirunyan et al. (CMS Collaboration), Phys. Rev. D 104, 032013 (2021).

[22] K.-F. Chen, W.-S. Hou, C. Kao, and M. Kohda, Phys. Lett. B 725, 378 (2013).

[23] W.-S. Hou, R. Jain, C. Kao, M. Kohda, B. McCoy, and A. Soni, Phys. Lett. B 795, 371 (2019).

[24] A. Abdesselam et al. (Belle Collaboration), arXiv:2103 .12994.

[25] A. M. Sirunyan et al. (CMS Collaboration), J. High Energy Phys. 03 (2020) 103.

[26] S. Davidson and H. E. Haber, Phys. Rev. D 72, 035004 (2005).

[27] W.-S. Hou and T. Modak, Phys. Rev. D 101, 035007 (2020).

[28] W.-S. Hou and T. Modak, Phys. Rev. D 103, 075015 (2021).

[29] D. Eriksson, J. Rathsman, and O. Stål, Comput. Phys. Commun. 181, 833 (2010).

[30] Having checked this, we set $\rho_{\tau \tau}$ to zero in our numerical analysis for simplicity.

[31] D. Chang, W.-S. Hou, and W.-Y. Keung, Phys. Rev. D 48, 217 (1993).

[32] B. Altunkaynak, W. S. Hou, C. Kao, M. Kohda, and B. McCoy, Phys. Lett. B 751, 135 (2015).

[33] W.-S. Hou and G. Kumar, Phys. Rev. D 102, 115017 (2020).

[34] D. K. Ghosh, W.-S. Hou, and T. Modak, Phys. Rev. Lett. 125, 221801 (2020).

[35] R. Glattauer et al. (Belle Collaboration), Phys. Rev. D 93, 032006 (2016).

[36] M. Kohda, T. Modak, and W.-S. Hou, Phys. Lett. B 776, 379 (2018).

[37] K. Hayasaka, K. Inami, Y. Miyazaki et al. Phys. Lett. B 687 , 139 (2010).

[38] A. Crivellin, A. Kokulu, and C. Greub, Phys. Rev. D 87, 094031 (2013).

[39] A. M. Sirunyan et al. (CMS Collaboration), Phys. Lett. B 798, 134992 (2019). 
[40] Y. S. Amhis et al. (HFLAV Collaboration), Eur. Phys. J. C 81, 226 (2021).

[41] M. Buschmann, J. Kopp, J. Liu, and X.-P. Wang, J. High Energy Phys. 06 (2016) 149.

[42] W.-S. Hou, M. Kohda, and T. Modak, Phys. Lett. B 798, 134953 (2019).

[43] A. M. Sirunyan et al. (CMS Collaboration), Eur. Phys. J. C 80, 75 (2020).

[44] T. Abe, R. Sato, and K. Yagyu, J. High Energy Phys. 07 (2017) 012.

[45] Y. Abe, T. Toma, and K. Tsumura, J. High Energy Phys. 06 (2019) 142.

[46] L. Wang and Y. Zhang, Phys. Rev. D 100, 095005 (2019).

[47] H.-X. Wang, L. Wang, and Y. Zhang, arXiv:2104.03242.

[48] C. W. Chiang, K. Fuyuto, and E. Senaha, Phys. Lett. B 762, 315 (2016).

[49] H.-K. Guo, Y.-Y. Li, T. Liu, M. Ramsey-Musolf, and J. Shu, Phys. Rev. D 96, 115034 (2017).

[50] K. Fuyuto, W.-S. Hou, and E. Senaha, Phys. Lett. B 776, 402 (2018).
[51] G. Aad et al. (ATLAS Collaboration), Phys. Rev. Lett. 125, 051801 (2020).

[52] A. M. Sirunyan et al. (CMS Collaboration), J. High Energy Phys. 09 (2018) 007.

[53] N. Ghosh and J. Lahiri, Phys. Rev. D 103, 055009 (2021).

[54] R. Primulando, J. Julio, and P. Uttayarat, Phys. Rev. D 101, 055021 (2020).

[55] See e. g. A. Dedesand H. E. Haber, J. High Energy Phys. 05 (2001) 006.

[56] S. M. Barr and A. Zee, Phys. Rev. Lett. 65, 21 (1990).

[57] V. Ilisie, J. High Energy Phys. 04 (2015) 077, and references therein.

[58] The sign of $\rho_{t t} \rho_{\mu \mu}$ needs to be negative for generating muon $g-2$ by a pure two-loop mechanism. But the rather large $\rho_{\mu \mu}$ would have its own one-loop effect, where one can play with cancellations. We do not get into this, but deem a purely two-loop mechanism as not viable.

[59] M. Aaboud et al. (ATLAS Collaboration), J. High Energy Phys. 07 (2019) 117. 